\title{
Lipid mediator profiles differ between lung compartments in asthmatic and healthy humans
}

\author{
Nirina Larsson1, Susanna L. Lundström², Rui Pinto3,4, Gregory Rankin, \\ Masoumeh Karimpour ${ }^{3}$, Anders Blomberg', Thomas Sandström', \\ Jamshid Pourazar', Johan Trygg ${ }^{3}$, Annelie F. Behndig' ${ }^{1}$, Craig E. Wheelock ${ }^{2}$ \\ and Malin L. Nording ${ }^{3}$
}

Affiliations: 'Dept of Public Health and Clinical Medicine, Division of Medicine, Umeå University, Umeå, ${ }^{2}$ Dept of Medical Biochemistry and Biophysics, Division of Physiological Chemistry II, Karolinska Institutet, Stockholm, ${ }^{3}$ Computational Life Science Cluster, Dept of Chemistry, Umeå University, Umeå, and ${ }^{4}$ Bioinformatics Infrastructure for Life Sciences, Linköping, Sweden.

Correspondence: C.E. Wheelock, Dept of Medical Biochemistry and Biophysics, Division of Physiological Chemistry II, Karolinska Institutet, Scheeles väg 2, SE-171 77, Stockholm, Sweden. E-mail: Craig. Wheelockaki.se

ABSTRACT Oxylipins are oxidised fatty acids that can exert lipid mediator functions in inflammation, and several oxylipins derived from arachidonic acid are linked to asthma. This study quantified oxylipin profiles in different regions of the lung to obtain a broad-scale characterisation of the allergic asthmatic inflammation in relation to healthy individuals.

Bronchoalveolar lavage fluid (BALF), bronchial wash fluid and endobronchial mucosal biopsies were collected from 16 healthy and 16 mildly allergic asthmatic individuals. Inflammatory cell counts, immunohistochemical staining and oxylipin profiling were performed. Univariate and multivariate statistics were employed to evaluate compartment-dependent and diagnosis-dependent oxylipin profiles in relation to other measured parameters.

Multivariate modelling showed significantly different bronchial wash fluid and BALF oxylipin profiles in both groups $\left(\mathrm{R}^{2} \mathrm{Y}[\mathrm{cum}]=0.822\right.$ and $\left.\mathrm{Q}^{2}[\mathrm{cum}]=0.759\right)$. Total oxylipin concentrations and five individual oxylipins, primarily from the lipoxygenase (LOX) pathway of arachidonic and linoleic acid, were elevated in bronchial wash fluid from asthmatics compared to that from healthy controls, supported by immunohistochemical staining of 15-LOX-1 in the bronchial epithelium. No difference between the groups was found among BALF oxylipins.

In conclusion, bronchial wash fluid and BALF contain distinct oxylipin profiles, which may have ramifications for the study of respiratory diseases. Specific protocols for sampling proximal and distal airways separately should be employed for lipid mediator studies.

@ERSpublications

Distinct oxylipin profiles of different areas of the lung and potential ramifications for the study of respiratory disease http://ow.ly/rmQZm

This article has supplementary material available from www.erj.ersjournals.com

Received: Dec 312012 | Accepted after revision: June 132013 | First published online: Sept 132013

Support statement: The Swedish Research Council Formas (Stockholm, Sweden) and the Swedish Heart-Lung Foundation (Stockholm) are gratefully acknowledged for their financial support. S.L. Lundström was funded by the Bernard Osher Initiative for Research on Severe Asthma (Stockholm). C.E. Wheelock was funded by the Centre for Allergy Research and the Karolinska Institutet (Stockholm).

Conflict of interest: None declared.

Copyright @ERS 2014 


\section{Introduction}

Asthma is a common respiratory disease characterised by chronic airway inflammation, bronchial hyperresponsiveness and airflow limitation which affects $\sim 300$ million people worldwide with a substantial disease burden in terms of morbidity, mortality and economic cost $[1,2]$. There is a broad range of asthmatic phenotypes with different clinical presentation and pathophysiology [3].

The role of lipid mediators in the pathogenesis of asthma has been extensively studied [4-10]. Lipid mediators are included in a group of compounds broadly called oxylipins, a term that includes eicosanoids derived from arachidonic acid as well as compounds derived from other related $\omega-3$ and $\omega-6$ fatty acids. These compounds are produced via three biosynthetic pathways: lipoxygenase (LOX) (e.g. leukotrienes), cyclo-oxygenase (e.g. prostaglandins) and cytochrome P450 (CYP) [11, 12]. In particular, 15-LOX expression and induction have been shown to be elevated in the lower airways of asthmatics [13, 14]. Both pro- and anti-inflammatory characteristics have been attributed to 15-LOX lung activity, in the form of derivatives such as eoxins (pro-inflammatory) and lipoxins (anti-inflammatory), as well as through the protective $\omega-3$ derived resolvins and protectins [12, 15-17]. The dual properties of 15-LOX activity illustrate how complex biological interactions involving multiple oxylipin species affect the initiation, progression and resolution of inflammation.

Oxylipin profiles have previously been investigated in large-volume bronchoalveolar lavage fluid (BALF), mainly reflecting profiles in the peripheral lung [7,8], but not in small-volume bronchial wash samples, which better reflect the proximal airways. To test the hypothesis that asthmatics, due to their underlying asthmatic inflammation, have altered oxylipin profiles in both distal (BALF) and proximal (bronchial wash) regions of the lung compared with healthy individuals, bronchoscopies were performed. A broad selection of compounds was quantified in both BALF and bronchial wash fluid through an oxylipin metabolic profiling approach. The resulting profiles were investigated together with clinical parameters and inflammatory cell counts using univariate and multivariate statistics, highlighting the utility of broad-scale metabolic profiling methods to investigate respiratory disease.

\section{Materials and methods}

Subjects

Healthy and asthmatic subjects were invited to participate in the present study through advertisements. Subjects fulfilled the following inclusion criteria: age 18-40 years, never-smokers, normal lung function (forced expiratory volume in $1 \mathrm{~s}$ (FEV1) and forced vital capacity (FVC) of $\geqslant 80 \%$ predicted and a normal FEV1/FVC ratio) and absence of concomitant diseases, apart from allergy in the asthmatic group. All asthmatics had a positive history of allergy together with at least one positive skin prick test against a standard panel of common aeroallergens. In the asthmatic group, bronchial hyperresponsiveness to methacholine with provocative concentration causing a $20 \%$ fall in FEV $1<8 \mathrm{mg} \cdot \mathrm{mL}^{-1}$ was required. All participants were free of respiratory infection for 6 weeks prior to the study. No antioxidant supplementation or anti-inflammatory medication was allowed for 2 weeks prior to the study. Asthma severity was classified as intermittent according to Global Initiative for Asthma guidelines [18], treated only with short-acting inhaled $\beta_{2}$-agonists on demand. Inhaled or nasal corticosteroids were terminated $\geqslant 3$ months prior to the study. 32 Caucasian volunteers were included: 16 asthmatics and 16 healthy controls. Subject demographics are shown in table 1.

Three satisfactory lung function measurements were performed, according to the recommendations of the American Thoracic Society (ATS) [19], using a Vitalograph spirometer (Vitalograph Ltd, Maids Moreton, UK). Methacholine challenge was performed using the method described by JUNIPER et al. [20].

\section{TABLE 1 Characteristics of the study population}

\section{Asthmatics}

Healthy controls

Subjects
Male/female
Age years
BMI $\mathrm{kg} \cdot \mathrm{m}^{-2}$
FEV1 \% pred
Methacholine PC20 $\mathrm{mg} \cdot \mathrm{mL}^{-1}$
Skin prick test

Data are presented as $n$, mean \pm SD or median (interquartile range). BMI: body mass index; FEV1: forced expiratory volume in $1 \mathrm{~s}$; \% pred: \% predicted; PC20: provocative concentration causing a $20 \%$ fall in FEV1. 
Informed consent was obtained from all volunteers after administering verbal and written information. The study was approved by the local ethics review board at Umeå University, Umeå, Sweden, and performed according to the Declaration of Helsinki.

\section{Study design}

The study was performed outside the pollen season. Prior to the study visit, subjects fasted from midnight and were asked to refrain from alcohol-containing beverages for $24 \mathrm{~h}$. Measurements of exhaled nitric oxide fraction $(\mathrm{FeNO})$ and bronchoscopy were performed in the morning. Healthy and asthmatic participants were examined in a randomised order.

\section{Exhaled nitric oxide fraction}

Before measurements, the participants rinsed their mouths with water. The participants wore a nose-clip while first inhaling deeply and then slowly exhaling against a resistance according to ATS/European Respiratory Society recommendations [21]. FeNO was measured three times at each flow rate (10, 50 and $100 \mathrm{~mL} \cdot \mathrm{s}^{-1}$ ) using a chemiluminescence analyser (NiOX; Aerocrine AB, Stockholm, Sweden).

\section{Bronchoscopy}

Bronchoscopy was performed as previously described [22], using a flexible video bronchoscope (Olympus BF-1T160; Olympus, Tokyo, Japan). A detailed description of the method is provided in the online supplementary material. Endobronchial mucosal biopsies were taken from proximal cristae. Bronchial wash $(2 \times 20 \mathrm{~mL})$ and bronchoalveolar lavage $(3 \times 60 \mathrm{~mL})$ were performed with saline solution on the contralateral side. The aspirates recovered from the instillations of bronchial wash and bronchoalveolar lavage were processed and analysed as previously described [23].

\section{Immunohistochemistry}

Endobronchial mucosal biopsies were processed and embedded into glycol methacrylate resin (Polyscience, Northampton, UK), according to the method developed by BRITTEN et al. [24]. 2- $\mu$ m-thick sections were cut and stained immunohistochemically. The monoclonal antibodies used were directed against mast cells, eosinophils, neutrophils and 15-LOX-1. The immunostaining procedure has been described previously [23]. Stained inflammatory cells were counted in the epithelium and in the submucosa, excluding glands, blood vessels and muscle. The cell counts were expressed as cells $\cdot \mathrm{mm}^{-1}$ in the epithelium and cells $\cdot \mathrm{mm}^{-2}$ in the submucosa and counted using a light microscope. 15-LOX-1 staining was quantified and expressed as percentage of stained epithelial area. Length and areas of the epithelium and submucosa and positive epithelial staining areas were determined using the programme LeicaQWin V3 (Leica Microsystems, Wetzlar, Germany).

\section{Oxylipin extraction and analysis}

Oxylipin analysis was performed according to previously published protocols $[25,26]$. Standards (native and deuterated) and $N$-cyclohexyl- $N^{\prime}$-dodecanoic acid urea used for recovery calculations were obtained from Cayman Chemical (Ann Arbor, MI, USA), Larodan Fine Chemicals AB (Malmö, Sweden) and Biomol International (Plymouth Meeting, PA, USA). The list of analysed oxylipins is given in online supplementary table S1. Solid phase extraction was performed using Oasis HBL $60 \mathrm{mg}$ cartridge columns (Waters, Milford, MA, USA). Oxylipins were analysed using an Acquity ultraperformance liquid chromatography separation module (Waters), equipped with a $2.1 \times 150-\mathrm{mm}$ BEH C18 column with a $1.7-\mu \mathrm{m}$ particle size (Waters) coupled to a Xevo TQ triple quadrupole tandem mass spectrometer (Waters) run in negative ionisation mode. Quantification of oxylipins above the limit of quantification (LOQ), defined as peaks with signal to noise ratio $(\mathrm{S} / \mathrm{N})>10$, was performed using the stable isotope dilution method. Oxylipins below the LOQ, but above the limit of detection (LOD), defined as peaks with $\mathrm{S} / \mathrm{N}>3$, were assigned the method LOD value in the subsequent statistical analyses.

\section{Statistical analysis}

Univariate statistical analysis using Mann-Whitney U-test was performed using PASW Statistics 18.0 (SPSS, Chicago, IL, USA) or R software (R Foundation for Statistical Computing, Vienna, Austria). $\mathrm{p}<0.05$ was considered significant. Multivariate data analysis (MVA) using principal component analysis (PCA) and orthogonal projections to latent structures with discriminant analysis (OPLS-DA) was performed using SIMCA software v.13 (Umetrics AB, Umeå, Sweden) [27]. Model validity was assessed using the cumulative amount of systematic variation among the variables summarised by the model $\left(\mathrm{R}^{2} \mathrm{X}[\mathrm{cum}]\right.$ and $\left.\mathrm{R}^{2} \mathrm{Y}[\mathrm{cum}]\right)$, the predictive ability of the model $\left(\mathrm{Q}^{2}[\mathrm{cum}]\right)$, and p-values calculated by ANOVA based on the crossvalidated score vectors [28]. All data were scaled to unit variance and mean-centred prior to modelling. 
TABLE 2 Exhaled nitric oxide fraction ( $\mathrm{FeNO}$ ) in asthmatic and healthy individuals

Flow rate $\mathrm{mL} \cdot \mathrm{s}^{-1}$

FeNO ppb

\begin{tabular}{lccc}
\cline { 2 - 3 } & Asthmatics $^{\#}$ & Healthy subjects & p-value \\
\hline $\mathbf{1 0 0}$ & $12.8(7.4-26.0)$ & $8.3(6.5-10.3)$ & 0.050 \\
$\mathbf{5 0} \mathbf{1 0}$ & $20.3(11.2-48.3)$ & $14.1(9.9-17.1)$ & 0.053 \\
\hline
\end{tabular}

Data are presented as median (interquartile range), unless otherwise stated. Bold type represents statistical significance at $\mathrm{p}<0.05{ }^{*}: \mathrm{n}=16$.

A description of these multivariate methods is provided in the online supplementary material, of which a summary is provided here. MVA can be described as a tool for reducing the dimensionality of large datasets to render the visualisation and interpretation more manageable. The relationship between the clinical samples (observations) and collected metabolite data (variables) is described. PCA forms the basis of MVA, representing a multivariate data table as a low-dimensional plane, which provides an overview of the data. This overview may reveal groupings, trends and outliers. The first principal component (PC1) is the line in the metabolic space that best approximates all the data. Each sample is thereafter projected onto this PC line, which becomes its coordinate value along the line. This coordinate value for each sample is called a score. One PC line is usually insufficient to model the systematic variation of an entire metabolite dataset. Accordingly, a second PC (PC2) is calculated, which is orthogonal (perpendicular) to PC1. When two PCs have been derived, they together define a plane, or a "window", into the high-dimensional metabolite space that can be plotted as a scatterplot (PC1 versus PC2), termed a scores plot. There is a corresponding plot for the metabolites called the loadings plot. This plot reveals how the metabolites contribute to the structure of the scores plot, and can be used to link information between individual metabolites and clinical samples.

In its simplest form, OPLS-DA is used instead of PCA when additional sample knowledge exists (e.g. healthy versus asthmatics). OPLS-DA divides the variation in the data into two parts, one part that models the classseparating variance (or predictive variation) and another part that models the within-class separating variance (or orthogonal variation). OPLS can also be used to predict the class identity of unknown samples. For PCA and OPLS-DA models, the amount of modelled variation is defined as the goodness of fit $\left(\mathrm{R}^{2}\right)$. An $\mathrm{R}^{2}$ value of 1 indicates that all variation in the data is modelled, a value of 0 means that no variation in the data is modeled. The goodness of prediction $\left(\mathrm{Q}^{2}\right)$ is based on cross-validation. $\mathrm{Q}^{2}$ values of 1 reflect perfect predictive precision, while values equal to or below 0 indicate that a random guess is more accurate. These metrics are used to evaluate the quality of PCA and OPLS-DA models.

\section{Results}

Airway inflammation

FeNO was measured as a noninvasive marker of airway inflammation. Asthmatics showed apparent higher FeNO values at all flow rates, compared with healthy controls. However, significance was only reached at the $10-\mathrm{mL} \cdot \mathrm{s}^{-1}$ flow rate (table 2). Airway inflammation was further assessed using bronchoscopy to sample inflammatory cells from different regions of the lung. Median bronchoalveolar lavage recoveries (reflecting peripheral lung compartments) were $70 \%$ for asthmatics and $75 \%$ for healthy controls and median bronchial wash recoveries (reflecting more proximal compartments) were $39 \%$ and $45 \%$, respectively. There was no significant difference between recovered volumes between the two groups.

Eosinophils and mast cells were elevated in bronchial wash fluid from asthmatics compared to that from healthy individuals (table 3), with no differences between cell counts in BALF. Endobronchial mucosal biopsies revealed higher eosinophil and mast cell numbers in the asthmatic epithelium, while submucosal neutrophil numbers were higher in the healthy individuals (table 3 ).

\section{Oxylipin profiles}

Of the 88 oxylipins screened using liquid chromatography-tandem mass spectrometry, 17 were above the LOQ and used in subsequent univariate and multivariate statistical analyses. PCA analysis of the oxylipin data revealed a gross asthmatic outlier, which was removed. A simplified pathway map of oxylipins included in the statistical analyses together with relative amounts in bronchial wash fluid and BALF from asthmatics and healthy controls is shown in figure 1 . The complete list of the 88 screened oxylipins and acronym 
definitions along with values above LOD and LOQ is provided in online supplementary table S1. Notably, a common isoprostane (8-isoprostaglandin $\mathrm{E}_{2}$ ) was not detected in any of the samples.

The median oxylipin concentrations in bronchial wash fluid and BALF in both asthmatics and healthy individuals ranged over three orders of magnitude from $\sim 1 \mathrm{pM}$ to $1 \mathrm{nM}$ (table 4 ). Five bronchial wash fluid oxylipins (15-hydroxyeicosatetraenoic acid (HETE), 9-hydroxyoctadienoic acid (HODE), 13-HODE, 13-oxo-octadecadienoic acid (KODE) and 15-hydroxyeicosatrienoic acids (HETrE)), predominantly originating from the 15-LOX pathway and auto-oxidation, as well as total oxylipin levels in bronchial wash fluid, were significantly elevated in asthmatics versus healthy individuals (table 4). No BALF oxylipin levels differed significantly between asthmatics and healthy individuals. Comparison of the oxylipin levels in bronchial wash fluid versus BALF evidenced several differences in both asthmatic and healthy subjects (table 4). Generally, 15-LOX-derived lipids were greater in bronchial wash fluid and CYP-derived products greater in BALF, independent of disease. Levels of the 15-LOX products 12-HETE, 15-HETE, 15-oxoeicosatetraenoic acid, 15-HETrE and 13-hydroxyoctadecatrienoic acid (HOTE) were higher in bronchial wash fluid in both groups, while 13-HODE levels were higher in only the asthmatic subjects. Levels of the CYP-derived 9(10)-epoxyoctamonoenoic acid (EpOME) and 12(13)-EpOME were higher in BALF of both groups, while 9,10-dihydroxyoctamonoenoic acid (DiHOME) was only higher in asthmatics. The synthetic origin of the linoleic-derived 9,10,13-trihydroxyoctamonoenoic acids (TriHOME) and 9,12,13-TriHOME is unclear, but they are potentially products of a mixture of 15-LOX and CYP activity and/or auto-oxidation. However, TriHOME response mirrored that of the CYP-derived compounds, with 9,10,13-TriHOME higher in BALF of both groups and 9,12,13-TriHOME higher in the BALF of healthy individuals.

\section{5-lipoxygenase}

Bronchial biopsies were stained for 15-LOX-1, with staining observed only in the epithelium. The epithelial expression of 15-LOX-1 was higher in the asthmatics compared with the healthy controls; however, levels did not reach statistical significance $(\mathrm{p}=0.08)$ (fig. 2). OPLS modelling evidenced a correlation between

TABLE 3 Inflammatory cell counts in bronchial wash fluid, bronchoalveolar lavage fluid (BALF), submucosa and epithelium in asthmatic and healthy individuals

\begin{tabular}{|c|c|c|c|}
\hline Cell type and location & Asthmatics" & Healthy subjects" & p-value \\
\hline \multicolumn{4}{|c|}{ Bronchial wash fluid $\times 10^{4}$ cells $\cdot \mathrm{mL}^{-1}$} \\
\hline Macrophages & $5.3(4.6-9.2)$ & $7.2(5.2-12.3)$ & 0.070 \\
\hline Neutrophils & $0.2(0.1-0.6)$ & $0.7(0.3-1.0)$ & 0.059 \\
\hline Lymphocytes & $0.6(0.3-0.9)$ & $0.3(0.2-0.7)$ & 0.346 \\
\hline Eosinophils & $0.1(0.0-0.3)$ & $0.0(0.0-0.1)$ & 0.012 \\
\hline Mast cells & $0.03(0.01-0.04)$ & $0.01(0.00-0.02)$ & 0.013 \\
\hline \multicolumn{4}{|l|}{ BALF $\times 10^{4}$ cells $\cdot \mathrm{mL}^{-1}$} \\
\hline Macrophages & $12.0(10.6-16.1)$ & $13.9(8.9-17.6)$ & 0.598 \\
\hline Neutrophils & $0.1(0.0-0.2)$ & $0.1(0.0-0.2)$ & 0.663 \\
\hline Lymphocytes & $1.1(0.6-1.4)$ & $0.7(0.5-1.0)$ & 0.200 \\
\hline Eosinophils & $0.1(0.0-0.2)$ & $0.0(0.0-0.1)$ & 0.180 \\
\hline Mast cells & $0.02(0.01-0.04)$ & $0.01(0.00-0.03)$ & 0.127 \\
\hline \multicolumn{4}{|l|}{ Submucosa cells $\cdot \mathrm{mm}^{-2}$} \\
\hline $\mathrm{CD}^{+}$lymphocytes & $20.9(4.6-51.2)$ & $20.4(4.2-135.0)$ & 0.777 \\
\hline $\mathrm{CD}^{+}$lymphocytes & $15.5(0.8-45.2)$ & $5.7(0.0-23.5)$ & 0.251 \\
\hline $\mathrm{CD8}^{+}$lymphocytes & $18.7(2.0-33.4)$ & $5.1(0.0-39.6)$ & 0.515 \\
\hline Mast cells & $29.0(18.0-36.2)$ & $31.1(16.1-52.0)$ & 0.474 \\
\hline Neutrophils & $38.8(23.1-93.9)$ & $104.4(55.9-167.3)$ & 0.029 \\
\hline Eosinophils & $1.1(0.0-9.3)$ & $0.0(0.0-0.0)$ & 0.083 \\
\hline \multicolumn{4}{|l|}{ Epithelium cells $\cdot \mathrm{mm}^{-1}$} \\
\hline $\mathrm{CD}^{+}$lymphocytes & $3.7(0.0-6.0)$ & $1.3(0.0-6.6)$ & 0.538 \\
\hline CD4 ${ }^{+}$lymphocytes & $0.0(0.0-0.8)$ & $0.0(0.0-0.0)$ & 0.572 \\
\hline $\mathrm{CD}^{+}$lymphocytes & $5.4(0.0-6.9)$ & $3.5(0.0-8.4)$ & 0.599 \\
\hline Mast cells & $0.0(0.0-1.3)$ & $0.0(0.0-0.0)$ & 0.006 \\
\hline Neutrophils & $0.5(0.0-1.7)$ & $0.0(0.0-2.3)$ & 0.441 \\
\hline Eosinophils & $0.0(0.0-1.3)$ & $0.0(0.0-0.0)$ & 0.006 \\
\hline
\end{tabular}

Data are presented as median (interquartile range), unless otherwise stated. Data are not corrected for multiple hypothesis testing. Significance is indicated at the $\alpha=0.05$ level, giving 1.2 potential false positives for the 22 measured values. The Bonferroni corrected $p$-value for these data is $\mathrm{p}=0.002$. Bold type represents statistical significance. ${ }^{\#}: \mathrm{n}=16$. 


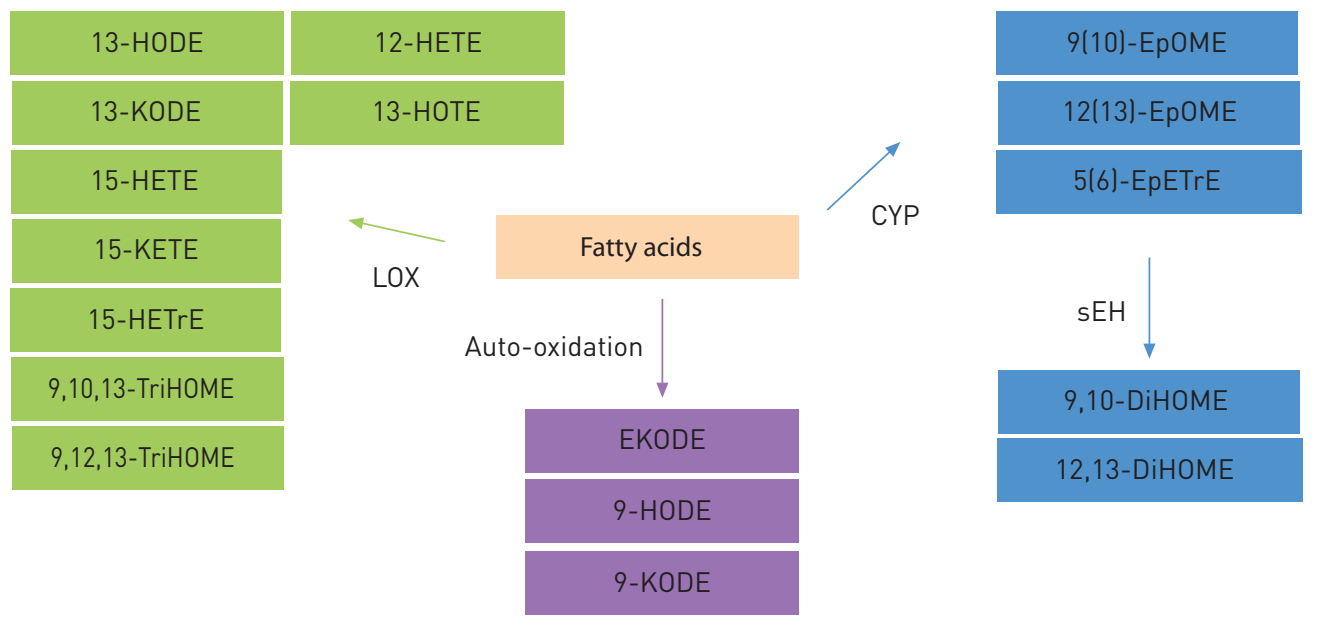

Asthmatic bronchial wash

Asthmatic BALF

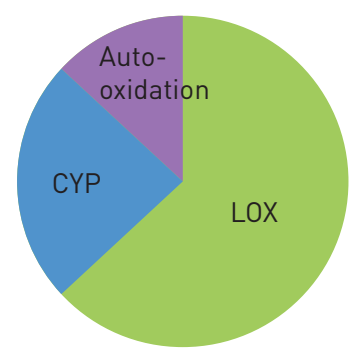

Healthy bronchial wash

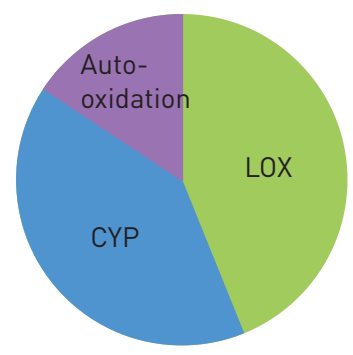

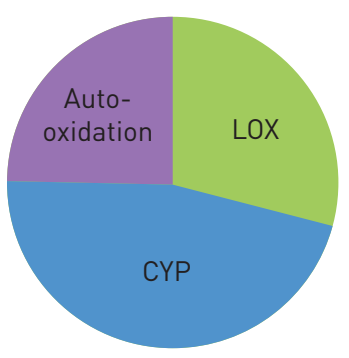

Healthy BALF

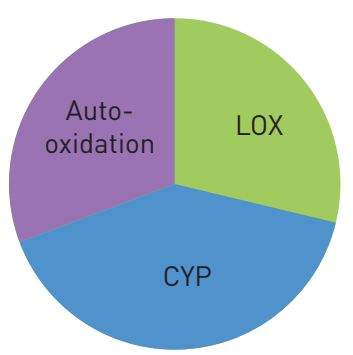

FIGURE 1 Schematic overview of oxylipins produced from fatty acid precursors via auto-oxidation, the lipoxygenase (LOX) and cytochrome P450 (CYP) enzymatic pathways, and their median relative contribution to the bronchial wash fluid and bronchoalveolar lavage fluid (BALF) profiles. The fatty acids linoleic acid, arachidonic acid, $\alpha$-linolenic acid and dihomo- $\gamma$-linolenic acid are precursors for hydroxyeicosatetraenoic acids (HETEs), hydroxyoctadienoic acids (HODEs), trihydroxyoctamonoenoic acids (TriHOMEs), hydroxyeicosatrienoic acids (HETrEs), hydroxyoctadecatrienoic acids (HOTEs), oxo-eicosatetraenoic acids (KETEs), oxo-octadecadienoic acids (KODEs), epoxyketo-octadecenoic acid (EKODE), epoxyeicosatrienoic acids (EpETrEs) and epoxyoctamonoenoic acids (EpOMEs), as well as the downstream soluble epoxide hydrolase (sEH) metabolites dihydroxyoctamonoenoic acids (DiHOMEs). Some of the oxylipins can be produced through alternative pathways, for instance 9-HODE, which also might be derived through the LOX pathway.

15-LOX-1 expression and oxylipin profile $\left(\mathrm{R}^{2} \mathrm{Y}=0.61, \mathrm{Q}^{2}[\mathrm{cum}]=0.471, \mathrm{p}=0.002\right)$ using data for 15-HETE, 9-HODE, 13-HODE, 13-KODE and 15-HETrE (significantly elevated in bronchial wash fluid from asthmatics compared to healthy controls) to predict 15-LOX-1 expression.

\section{Integrated multivariate modelling}

Multivariate statistical analysis was used to investigate patterns and relationships in the data. The component summarising the majority of the systematic variability was used in figure 3 to highlight the diagnosis-dependent and compartment-dependent variability of the profiles, respectively. The position of each subject along the $y$-axis was determined by its predicted OPLS-DA class definition value, based on 


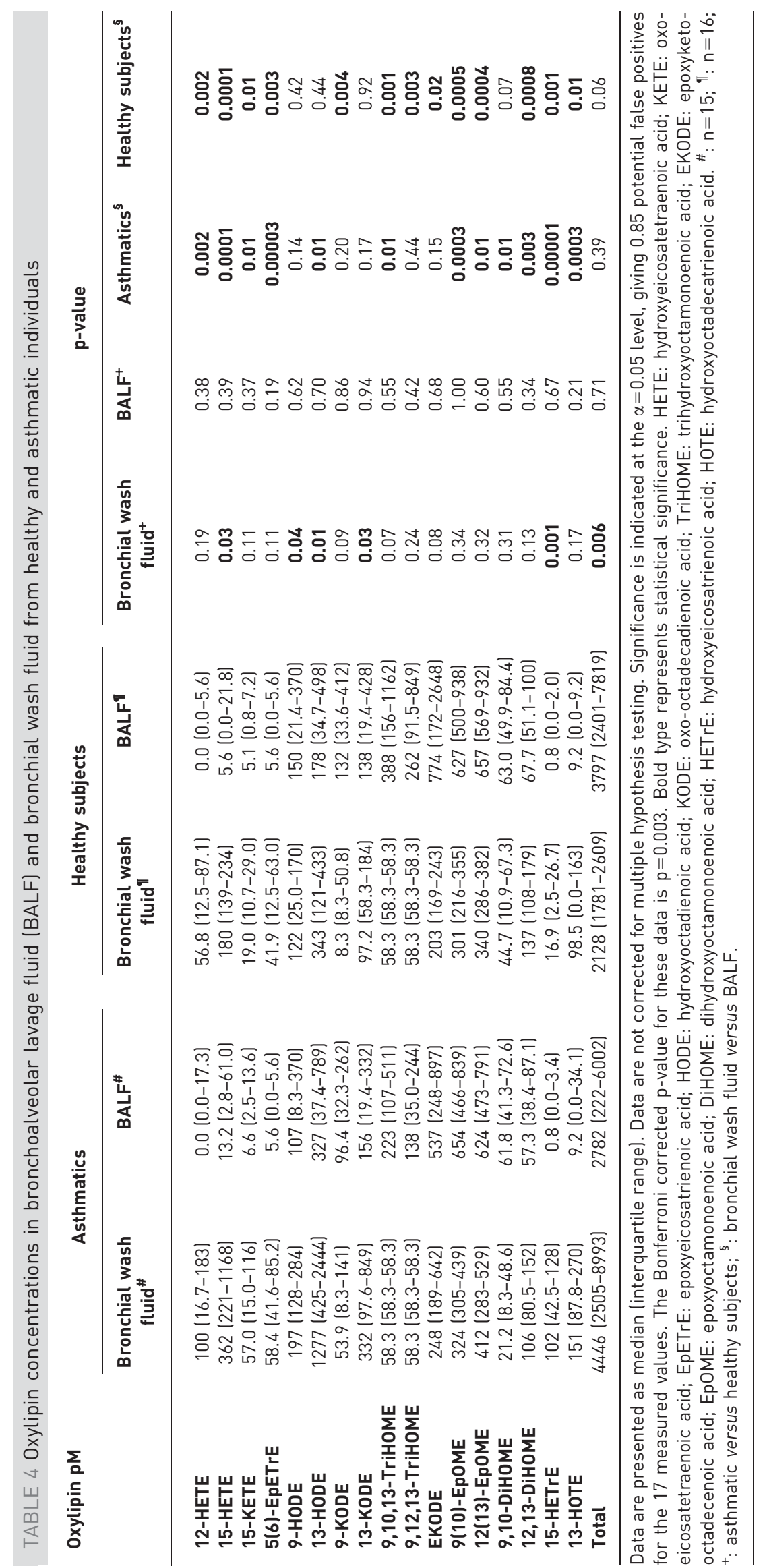



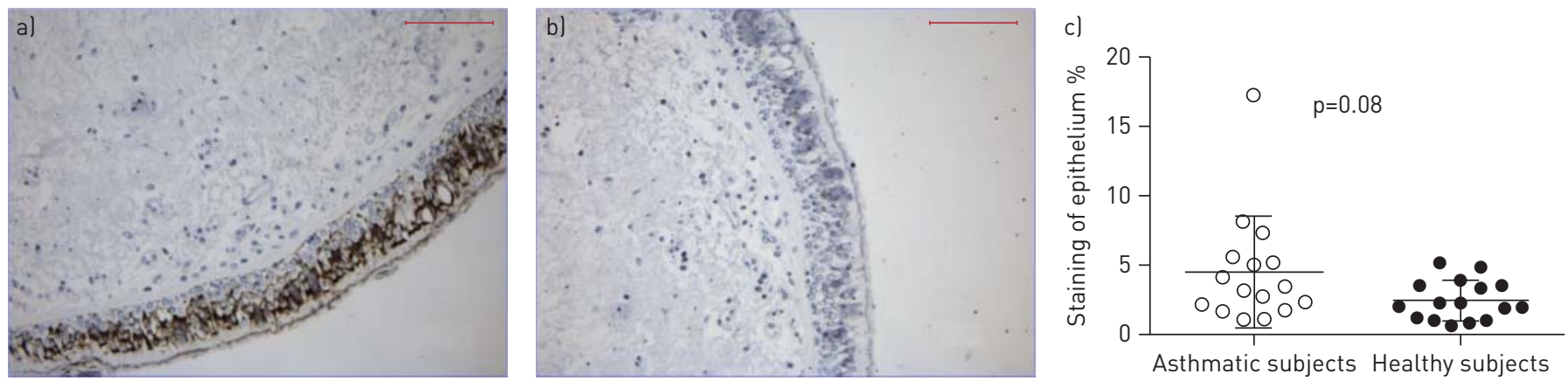

FIGURE 2 Representative pictures of endobronchial biopsies with a) 15-lipoxygenase (LOX)-1 staining compared with b) Tris-buffered saline control; c) fraction of epithelial area stained with $15-\mathrm{LOX}-1$ in asthmatics versus healthy controls. The staining was localised to the epithelium. Scale bars $=100 \mu \mathrm{m}$.

cross-validation calculations using each subject's profile of variables in relation to the rest of the subjects' profiles. Thereby, subjects with similar profiles (in the same class) clustered together either below or above 0.5, equivalent to the class definition threshold. See online supplementary table S2 for validation parameters.

An OPLS-DA model $\left(\mathrm{R}^{2} \mathrm{Y}[\mathrm{cum}]=0.419\right.$ and $\left.\mathrm{Q}^{2}=0.241\right)$ for all collected data (demographics, cell numbers, oxylipins, FeNO and 15-LOX staining) separated asthmatics and healthy subjects along the $y$-axis, except for six asthmatics $>0.5$, and one healthy subject $<0.5$ (fig. 3a). The information was summarised by a model component capturing $\sim 20 \%$ of the data variability related to diagnosis. The healthy individuals were more homogenously clustered along the $y$-axis than the asthmatic group, indicating that the asthmatics display a larger variation in the outcomes. There was no clustering with respect to sex.

To interpret the influence of each parameter on the different profiles displayed by asthmatics and healthy subjects, loading values (i.e. the individual contribution from each parameter to the class separation) were calculated (online supplementary table S3). Variables with large absolute loading values and small confidence intervals were the main contributors to the diagnosis-dependent profiles, while variables close to zero displayed nonsignificant influence. Variables with large negative values were typical for the asthmatic group and variables with large positive values were typical for the healthy group. In accordance with the univariate analysis, the major asthma drivers were $\mathrm{FeNO}$ and eosinophils in the epithelium together with the oxylipins 13-HODE, 13-KODE and epoxyketo-octadecenoic acid in bronchial wash fluid. Age and body mass index were among the variables with little or no influence on the diagnosis.

An OPLS-DA model of the oxylipin profiles displayed clustering with respect to lung compartment (fig. 3b). $\sim 20 \%$ of the oxylipin data were related to lung compartment. The intersubject variation was similar in both bronchial wash fluid and BALF, as well as among the asthmatic and healthy subjects, indicating that the baseline difference between bronchial wash fluid and BALF oxylipin profiles exists in both asthmatic and healthy subjects. Consequently, there was a relationship between separate OPLS-DA oxylipin models for asthmatics and healthy subjects, displayed in a shared and unique structure (SUS) plot $\left(\mathrm{R}^{2}=0.83\right)$ (fig. 4). See LUNDSTRÖM et al. [8] for an in-depth discussion on interpreting SUS plots. Oxylipin profiles in asthmatics ( $x$-axis) versus healthy individuals ( $y$-axis) revealed the same characteristic oxylipins for bronchial wash fluid (e.g. 13-HOTE and 5(6)-epoxyeicosatrienoic acid in the lower left corner of fig. 4), as well as for BALF (e.g. 9(10)-EpOME and 12(13)-EpOME in the upper right corner of fig. 4). Hence the difference in oxylipin profiles between bronchial wash fluid and BALF was largely independent of diagnosis. The class prediction and loading plots for the two different models are shown in online supplementary figures S1 (asthmatics) and S2 (healthy subjects).

\section{Discussion}

Oxylipin baseline profiles differed between proximal and distal airways in both asthmatics and healthy individuals, indicating lung region-specific lipid mediator production. These regional differences in oxylipin levels were further supported by allergic airway inflammation in terms of higher eosinophil and mast cell counts in the bronchial wash fluid and bronchial epithelium of asthmatics, compared with those of healthy controls, with no significant alterations in BALF. The asthmatics also expressed higher FeNO levels at $10 \mathrm{~mL} \cdot \mathrm{s}^{-1}$ than the healthy controls. These data suggest that enhanced lipid mediator production is associated with allergic asthmatic airway inflammation, predominantly in the proximal airways.

While auto-oxidation and CYP products were more common in BALF, the majority of the significantly elevated oxylipins in bronchial wash fluid from asthmatics were derived from the 15-LOX pathway of 

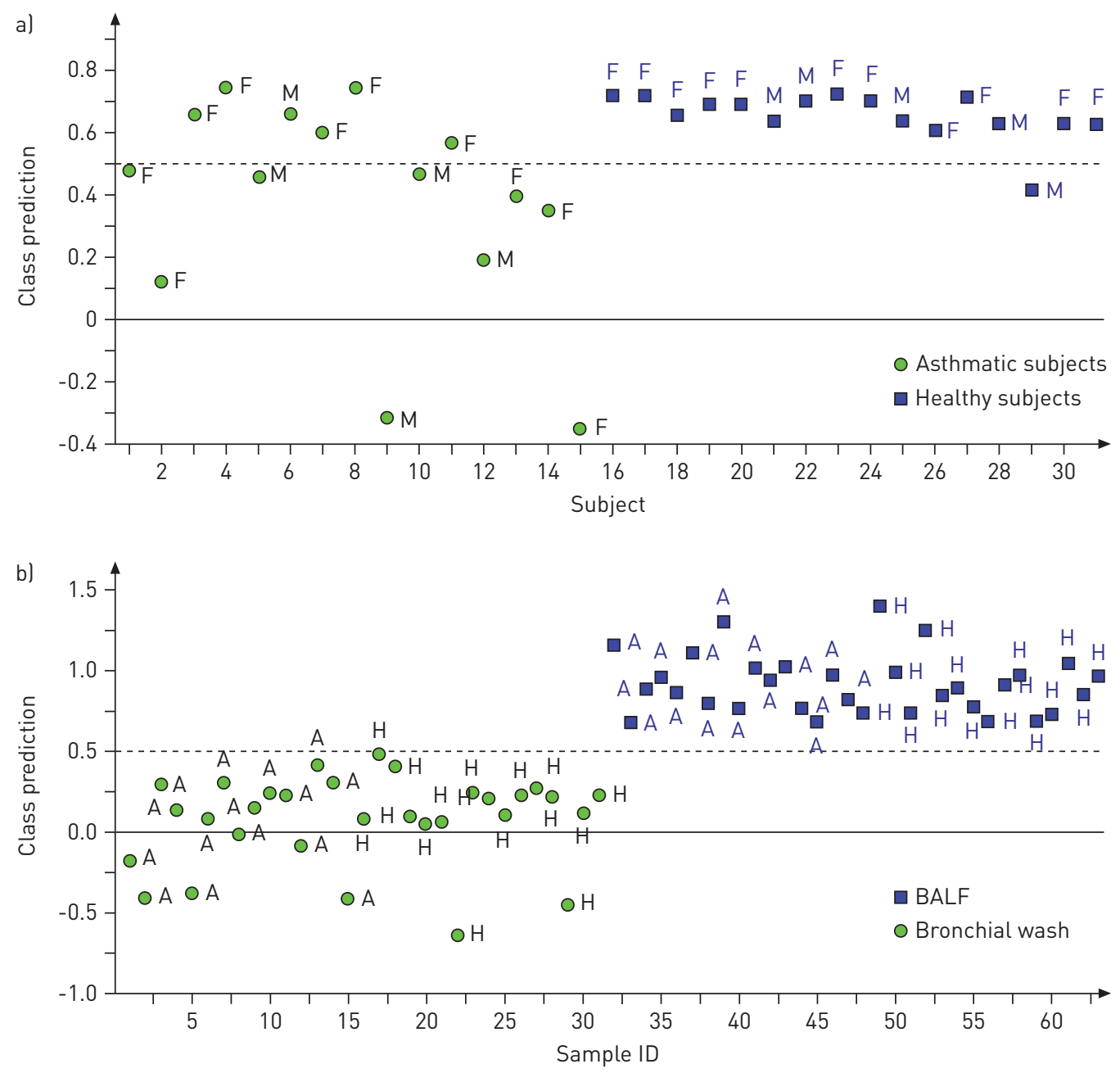

FIGURE 3 a) Diagnosis-dependent and b) compartment-dependent cross-validated orthogonal projections to latent structures with discriminant analysis class definition values. In the diagnosis-dependent model (a), asthmatics and healthy controls showed different profiles based on demographics, cell numbers, exhaled nitrogen oxide fraction, 15-lipoxygenase staining and oxylipins, resulting in different class definition values ( $<0.5$ for asthmatics and $>0.5$ for healthy subjects), except for six asthmatic subjects with similar profiles to the healthy group $(>0.5)$ and one healthy subject with a similar profile to the asthmatic group $(<0.5)$. Intersubject variation was larger in the asthmatic group than in the healthy group, while male $(\mathrm{M})$ and female $(\mathrm{F})$ subjects showed similar intersubject variation in both groups. In the compartmentdependent model (b), bronchoalveolar lavage fluid (BALF) and bronchial wash fluid showed different oxylipin profiles, resulting in different class definition values $(<0.5$ for bronchial wash fluid and $>0.5$ for BALF). Intersubject variation was similar in both bronchial wash fluid and BALF, as well as within the asthmatic (A) and healthy $(\mathrm{H})$ groups.

arachidonic and linoleic acid. These findings were supported by: 1) immunohistochemical data from stained endobronchial mucosal biopsies, and 2) multivariate correlations between 15-LOX data predicted by the five oxylipins elevated in bronchial wash fluid and 15-LOX-1 levels. Leukotoxin and iso-leukotoxin (9(10)-EpOME and 12(13)-EpOME) were among the most prominent species in the BALF oxylipin profile. These compounds, and their corresponding diols (9,10-DiHOME and 12,13-DiHOME; leukotoxin-diol and iso-leukotoxin-diol, respectively) are produced in leukocytes, and previous studies have shown that BALF levels of leukotoxin are elevated in respiratory distress syndrome [29]. Significantly elevated levels of 12,13-DiHOME have also been found in exhaled breath condensate from an asthmatic individual after allergen exposure [30]. Accordingly, these CYP products and their downstream soluble epoxide hydrolase metabolites are potentially of interest in the pathology of respiratory disease and merit further study.

Asthma-specific shifts in lipid mediators were only observed in the proximal lung regions, sampled through collection of bronchial wash fluid, in contrast to previous findings showing baseline asthma-related oxylipin alterations in BALF [8]. These seemingly contradictory findings most probably demonstrate the impact of employing different lavage protocols for airway sampling. The aim of the present study was to address 


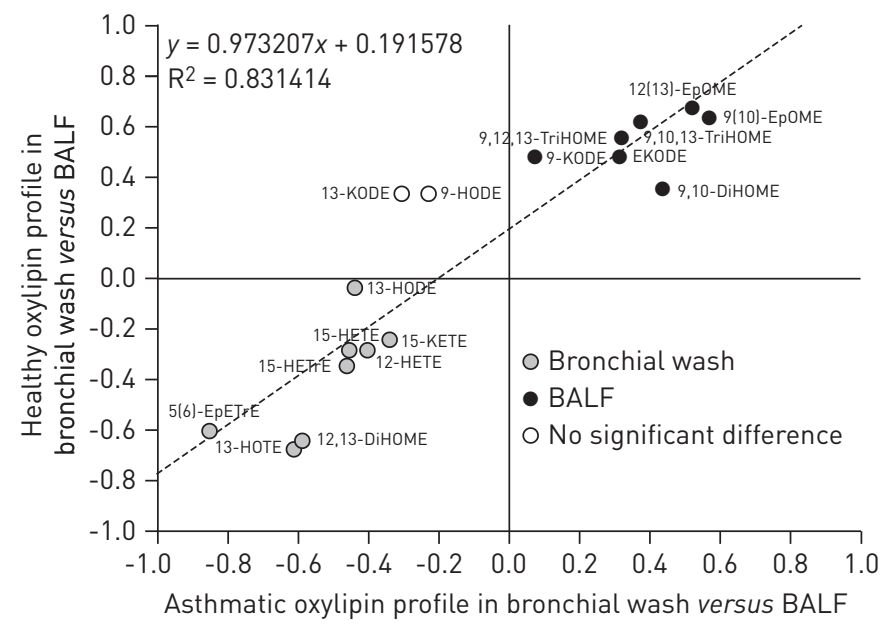

FIGURE 4 Shared and unique structure plot of two separate orthogonal projections to latent structures with discriminant analysis models for oxylipin profiles in asthmatic and healthy subjects. Oxylipins were correlated to bronchial wash fluid and bronchoalveolar lavage fluid (BALF) to the same extent in both models $\left(\mathrm{R}^{2}=0.83\right)$. Only 13 -oxo-octadecadienoic acid (KODE) and 9-hydroxyoctadienoic acid (HODE) were not significantly different between bronchial wash fluid and BALF in either of the two groups $(\mathrm{p}=0.05)$. EpETrE: epoxyeicosatrienoic acid; HOTE: hydroxyoctadecatrienoic acid; DiHOME: dihydroxyoctamonoenoic acid; HETrE: hydroxyeicosatrienoic acid; HETE: hydroxyeicosatetraenoic acids; KETE: oxo-eicosatetraenoic acid; EKODE: epoxyketo-octadecenoic acid; TriHOME: trihydroxyoctamonoenoic acid; EpOME: epoxyoctamonoenoic acid.

oxylipin levels in different lung compartments. Towards that end, a separate bronchial wash collection step was performed, in contrast to previous studies [8], in which the lavage procedure was not designed to study the difference between proximal and distal airways. In the current study, bronchial wash $(2 \times 20 \mathrm{~mL})$ and bronchoalveolar lavage $(3 \times 60 \mathrm{~mL})$ were performed sequentially using saline solution, while in the previous study bronchoalveolar lavage $(5 \times 50 \mathrm{~mL})$ was performed using PBS solution. Accordingly, the lack of observed differences in BALF between asthmatics and healthy individuals in the current study is attributed to the respective oxylipins being removed in the initial bronchial wash, whereas previous studies contained these oxylipin species in the BALF. These results emphasise the importance of well-defined lavage sampling protocols and the need for rigorous descriptions of the sampling methods. In addition, the findings demonstrate the added benefit in distinguishing different lung compartments provided by a sequential lavage procedure in which bronchial wash fluid and BALF are collected separately.

We based our hypothesis on the assumption that airway lavages reflecting different regions of the lung would reveal asthma-related oxylipin-specific attributes due to the underlying asthmatic inflammation in connection to oxidative stress. While this was true for bronchial wash, it was not the case for bronchoalveolar lavage in this particular study group of mildly allergic asthmatics. Previous studies using differentiated lavage volumes have given support to the notion that the asthmatic inflammation is more localised in the proximal airways and better sampled by small-volume bronchial wash, than the limited events occurring in the alveolar region, which is mostly sampled by a large-volume bronchoalveolar lavage [31].

The oxylipin composition of bronchial wash fluid and BALF differ, which has potential physiological implications for the study of asthma. The majority of oxylipin metabolic profiling work performed to date has focused on BALF; however, our results demonstrate that bronchial wash fluid provides a distinct oxylipin signature, which highlights the physiological implications of compartment-dependent sampling. Given that asthma is a disease primarily of the proximal airways, the sampling of bronchial wash fluid fluid may provide increased insight into the disease. In addition, asthma pathophysiology is linked to oxidative stress and lipid peroxidation, with isoprostanes often employed as oxidative markers [9]. While 8-isoprostaglandin- $\mathrm{E}_{2}$ (a common isoprostane) was not detected, we found elevated levels of five 15-LOX/ auto-oxidation derived oxylipins in bronchial wash fluid from asthmatics compared to healthy subjects. It is purported that reactive oxygen species are generated as by-products during conversion of the intermediate 15-LOX peroxide metabolites by glutathione reductase (e.g. formation of 15-HETE or 13-HODE) [32], which could subsequently affect the oxidative environment. It would therefore be of interest to explore whether differences in LOX metabolism affect the oxidative environment and associate with disease on a lung compartment-specific basis. Taken together, our findings illustrate the value of metabolic profiling of 
lipid mediators in respiratory disease and suggest that the effects of lung compartment-specific sampling should be considered in experimental design.

\section{Acknowledgements}

The authors wish to thank A-B. Lundström, S. Wennberg, E. Roos-Engstrand, A. Johansson and F. Holmström (Dept of Public Health and Clinical Medicine, Division of Medicine, Umeå University, Umeå, Sweden) for their expert technical assistance to this project. The authors would also like to thank the volunteers participating in this study.

\section{References}

Braman SS. The global burden of asthma. Chest 2006; 130: Suppl. 1, 4S-12S.

2 Masoli M, Fabian D, Holt S, et al. The global burden of asthma: executive summary of the GINA Dissemination Committee report. Allergy 2004; 59: 469-478.

Subbarao P, Mandhane PJ, Sears MR. Asthma: epidemiology, etiology and risk factors. CMAJ 2009; 181: E181-E190. Drazen JM, Israel E, O’Byrne PM. Treatment of asthma with drugs modifying the leukotriene pathway. $N$ Engl J Med 1999; 340: 197-206.

5 Lundström SL, Balgoma D, Wheelock ÅM, et al. Lipid mediator profiling in pulmonary disease. Curr Pharm Biotechnol 2011; 12: 1026-1052.

6 Haeggström JZ, Funk CD. Lipoxygenase and leukotriene pathways: biochemistry, biology, and roles in disease. Chem Rev 2011; 111: 5866-5898.

7 Lundström SL, Levänen B, Nording $M$, et al. Asthmatics exhibit altered oxylipin profiles compared to healthy individuals after subway air exposure. PLoS One 2011; 6: e23864.

8 Lundström SL, Yang J, Källberg HJ, et al. Allergic asthmatics show divergent lipid mediator profiles from healthy controls both at baseline and following birch pollen provocation. PLoS One 2012; 7: e33780.

9 Voynow JA, Kummarapurugu A. Isoprostanes and asthma. Biochim Biophys Acta 2011; 1810: 1091-1095.

10 Wheelock CE, Goss VM, Balgoma D, et al. Application of 'omics technologies to biomarker discovery in inflammatory lung diseases. Eur Respir J 2013; 42: 802-825.

11 Samuelsson B, Dahlén SE, Lindgren JA, et al. Leukotrienes and lipoxins: structures, biosynthesis, and biological effects. Science 1987; 237: 1171-1176.

12 Serhan CN, Petasis NA. Resolvins and protectins in inflammation resolution. Chem Rev 2011; 111: 5922-5943.

13 Chu HW, Balzar S, Westcott JY, et al. Expression and activation of 15-lipoxygenase pathway in severe asthma: relationship to eosinophilic phenotype and collagen deposition. Clin Exp Allergy 2002; 32: 1558-1565.

14 Shannon VR, Chanez P, Bousquet J, et al. Histochemical evidence for induction of arachidonate 15-lipoxygenase in airway disease. Am Rev Respir Dis 1993; 147: 1024-1028.

15 Feltenmark S, Gautam N, Brunnström A, et al. Eoxins are proinflammatory arachidonic acid metabolites produced via the 15-lipoxygenase-1 pathway in human eosinophils and mast cells. Proc Natl Acad Sci USA 2008; 105: 680-685.

16 Chiang N, Arita M, Serhan CN. Anti-inflammatory circuitry: lipoxin, aspirin-triggered lipoxins and their receptor ALX. Prostaglandins Leukot Essent Fatty Acids 2005; 73: 163-177.

17 Levy BD, Kohli P, Gotlinger K, et al. Protectin D1 is generated in asthma and dampens airway inflammation and hyperresponsiveness. J Immunol 2007; 178: 496-502.

18 Global Initiative for Asthma. GINA Report, Global Strategy for Asthma Management and Prevention. 2012. www. ginasthma.org/documents/4 Date last accessed: December 31, 2012. Date last updated: December 2012.

19 Standardization of Spirometry, 1994 Update. Am J Respir Crit Care Med 1995; 152: 1107-1136.

20 Juniper EF, Cockcroft DW, Hargreave FE. Histamine and Methacholine Inhalation Tests: Tidal Breathing Method: Laboratory Procedure and Standardisation. 2nd Edn. Lund, Astra Draco AB, 1994.

21 American Thoracic Society, European Respiratory Society. ATS/ERS recommendations for standardized procedures for the online and offline measurement of exhaled lower respiratory nitric oxide and nasal nitric oxide, 2005. Am J Respir Crit Care Med 2005; 171: 912-930.

22 Blomberg A, Mudway IS, Nordenhäll C, et al. Ozone-induced lung function decrements do not correlate with early airway inflammatory or antioxidant responses. Eur Respir J 1999; 13: 1418-1428.

23 Salvi S, Blomberg A, Rudell B, et al. Acute inflammatory responses in the airways and peripheral blood after shortterm exposure to diesel exhaust in healthy human volunteers. Am J Respir Crit Care Med 1999; 159: 702-709.

24 Britten KM, Howarth PH, Roche WR. Immunohistochemistry on resin sections: a comparison of resin embedding techniques for small mucosal biopsies. Biotech Histochem 1993; 68: 271-280.

25 Yang J, Schmelzer K, Georgi K, et al. Quantitative profiling method for oxylipin metabolome by liquid chromatography electrospray ionization tandem mass spectrometry. Anal Chem 2009; 81: 8085-8093.

26 Lundström SL, Saluja R, Adner M, et al. Lipid mediator metabolic profiling demonstrates differences in eicosanoid patterns in two phenotypically distinct mast cell populations. J Lipid Res 2013; 54: 116-126.

27 Trygg J, Wold S. Orthogonal projections to latent structures (O-PLS). J Chemometr 2002; 16: 119-128.

28 Eriksson L, Trygg J, Wold S. CV-ANOVA for significance testing of PLS and OPLS ${ }^{\mathrm{w}}$ models. J Chemometr 2008; 22: 594-600.

29 Ozawa T, Sugiyama S, Hayakawa M, et al. Existence of leukotoxin 9,10-epoxy-12-octadecenoate in lung lavages from rats breathing pure oxygen and from patients with the adult respiratory distress syndrome. Am Rev Respir Dis 1988; 137: 535-540.

30 Nording ML, Yang J, Hegedus CM, et al. Endogenous levels of five fatty acid metabolites in exhaled breath condensate to monitor asthma by high-performance liquid chromatography: electrospray tandem mass spectrometry. IEEE Sens J 2010; 10: 123-130.

31 Holgate ST. A look at the pathogenesis of asthma: the need for a change in direction. Discov Med 2010; 9: 439-447.

32 Kinder M. Fatty Acid Metabolism Mediated by 12/15-Lipoxygenase is a Novel Regulator of Hematopoietic Stem Cell Function and Myelopoiesis. Penn Dissertations, 2010. http://repository.upenn.edu/edissertations/88 Date last accessed: December 31, 2012. Date last updated: July 25, 2013. 